\title{
Characterization of Error Events and Design of a Robust Receiver for PRACH Detection
}

\author{
Francesco Linsalata $^{1 *}$, Maurizio Magarini ${ }^{1}$, Riccardo Ferrari ${ }^{2}$ \\ ${ }^{1}$ Politecnico di Milano, 20133 Milan, Italy \\ ${ }^{2}$ Azcom Technology s.r.l., 20089 Rozzano, Italy \\ (*corresponding author email: francesco.linsalata@polimi.com)
}

\begin{abstract}
In wireless cellular networks a user equipment can be scheduled for uplink transmission only if the timing is correctly synchronized. Hence, a dedicated channel must be established, i.e. the Physical Random-Access Channel (PRACH), which has a key role as interface between the nonsynchronized users' equipment and the uplink orthogonal transmission scheme. Due to the stringent requirements in terms of delay and influence of noise and multipath fading on the signal detection, the design of a robust PRACH receiver is a critical issue. Moreover, with the introduction of new type of services in fifth generation (5G) New Radio (NR) systems, such as ultra-reliable low-latency communications or machine-type communications, and with the need for mechanisms that are able to dynamically adapt to the changing environment, the design of a robust PRACH receiver is a priority but, at the same time, it opens the way to the development of new solutions. The performance of preamble detection in PRACH worsens when channels become more time dispersive. In order to guarantee an acceptable performance for most of users in a cell, in this paper we provide an improved PRACH receiver design that reduces the combined effect of different error events that determine the total missed detection probability. Considering the difficulty of achieving an accurate detection in some scenarios, a new robust three-step approach is proposed to overcome the issues of conventional PRACH signal detection. It is shown that the proposed approach outperforms the traditional peak detection one when the timedispersion of the channel increases. The benefits of the proposed solution are demonstrated by means of Monte Carlo simulations.
\end{abstract}

Keywords - PRACH, Zadoff-Chu Sequence, preamble detection, 5G, hypothesis testing.

\section{INTRODUCTION}

In Fourth (4G) and Fifth Generation (5G) standards for wireless cellular communications the Physical Random-Access Channel (PRACH) has the important role of establishing the initial uplink 


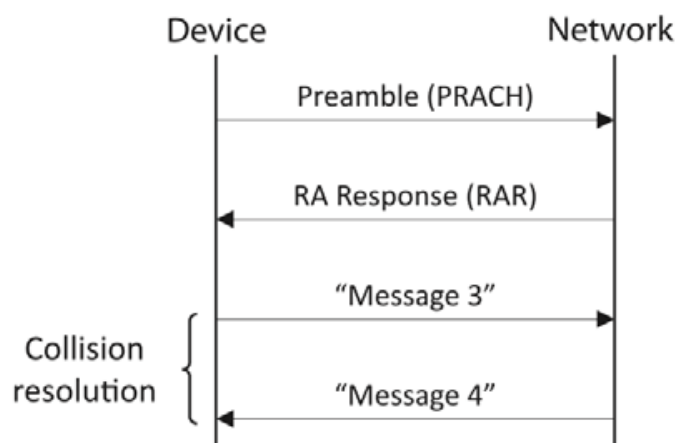

Figure 1. RACH procedure of LTE.

communication, including time synchronization and multiple access opportunity, from a User Equipment (UE) to the target Base Station (BS) [1]. In the initialization of a connection, a user first synchronizes with the target BS in the downlink by using its broadcasted information, which includes parameters such as PRACH preamble, target BS received preamble, and transmitted power. Then, based on the estimation of path loss and the open loop power control, users transmit a preamble on the PRACH channel satisfying the requirement of the BS target preamble received power. If the preamble is correctly detected without conflict, the user successfully establishes the uplink synchronization and builds the connection, after finishing subsequent interactions.

Long-Term Evolution (LTE) is based on the Random-Access Channel (RACH) procedure depicted in Fig. 1, which consists in:

1. transmission of a preamble, also referred as PRACH;

2. transmission of a Random-Access Response (RAR), which indicates the reception of the preamble and provides a time-alignment command to adjust the transmission timing of the UE;

3. uplink message;

4. downlink message, with the aim of resolving potential collisions due to simultaneous transmission of the same preamble from multiple devices within the cell.

Once the RACH procedure is complete, the device is in connected state and network-device communication can continue using normal dedicated transmission. Hence, we can define detection of the PRACH preamble signal as the first, and the most important, step in the RACH phase. In fact, if the PRACH procedure fails the user needs to resend the preamble after a predefined waiting time. This is also more relevant with the newest and more stringent latency requirements 
services required in 5G. A successful PRACH reception allows for the subsequent UE transmission to be inserted among the scheduled synchronized transmissions of other UEs, thus managing in a proper way the limited frequency-time resources. Moreover, this allows an accurate estimate of the Timing Advance (TA), which defines the time taken by the signal to reach the BS from the position of the UE. The continuous adaptation of the TA reduces the interference, minimizes the data loss, and maintains mobile Quality of Service (QoS).

Several approaches were proposed in the literature for the design of PRACH receivers in 4G longterm evolution (LTE) and LTE-Advanced (LTE-A) systems [2]-[5]. All these works consider as a reference against which to evaluate the performance a correct detection limit of at least $99 \%$, as outlined in the 3GPP 36.104 specifications [6]. The method proposed in this paper is based on the processing of the Power Delay Profile (PDP) of the received sequence with a three steps approach, where the samples are recombined and processed in each step. The idea is to take into account the different error events and how they affect the classical procedure. This has permitted us to justify the motivations and benefits of each new step. In particular, in the first step we developed a threshold adaptation approach considering the channel scenario, in the second one we filtered the PDP to remove the effect of the channel itself, and in the third, and last, step we took the decision about the peak position and TA.

The paper is organized as follows. Section II gives a short overview of the preamble sequence construction. Section III outlines the normal detection algorithm, whose issues and error events in real and practical implementation are commented in Sec. IV. Section V outlines the main problems of the classical approaches, while the proposed algorithm is described in Sec. VI. Simulation results are reported in Sec. VII. The proposed three-step PRACH signal detection, which is here considered in a 4G setup, can be easily extended to 5G New Radio (NR) as explained in the Sec. VIII.

Conclusions and forthcoming research activities are drawn in Sec. IX.

\section{PREAMBle SEQUENCE}

LTE and LTE-A are based on Orthogonal Frequency-Division Multiplexing Access (OFDMA) in the downlink. In the uplink Single-carrier Frequency-Division Multiple Access (SC-FDMA) is adopted. The preamble sequences used in LTE and LTE-A are Zadoff-Chu (ZC) sequences, which 
meet the required ideal auto-correlation property. In fact, it can be shown that ZC sequences satisfy the Constant Amplitude Zero Autocorrelation (CAZAC) property [7]. The prime ZC sequence is defined as:

$$
x_{u}(n)=e^{-\frac{j(\pi u n(n+1))}{N_{z c}}}, \quad 0 \leq n \leq N_{z c}-1,
$$

where $u$ is the root sequence number and $N_{z c}$ is a prime number. A ZC sequence is characterized by several properties, including the ideal cyclic autocorrelation one, which means that its autocorrelation is a Dirac delta function [7]. It is worth observing that sequences obtained from cyclic shifts of ZC sequences defined by two different roots are not orthogonal. Hence, orthogonal sequences obtained by cyclically shifting a single root sequence should be favored over nonorthogonal ones.

In LTE and LTE-A preamble sequences are built by cyclic shifts of a ZC sequence of prime length $N_{Z C}$. The cyclic shift offset $N_{C S}$ is chosen so that the Zero Correlation Zone (ZCZ) of the sequences, i.e. the number of samples between two peaks, guarantees the orthogonality inside the radius of the cell. In each LTE cell a number of 64 mutually orthogonal sequences are used, all obtained by cyclic shifts of the root sequence (see [8]) as follows:

$$
X_{u, v}=x_{u}\left(\left(n+C_{v}\right) \bmod N_{z c}\right) \text {, }
$$

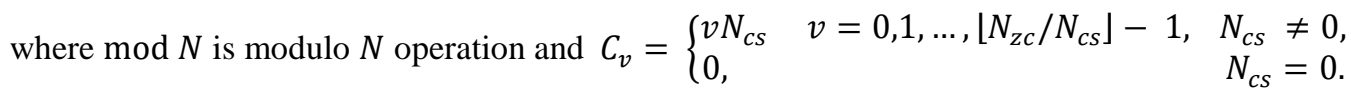

Note that ZC sequences with Cyclic Prefix (CP) are used for the construction of the PRACH preambles, where CP converts linear convolution to circular convolution), and ZC sequences keep the ideal circular autocorrelation property. Its projection is equidistant to any other sequence with

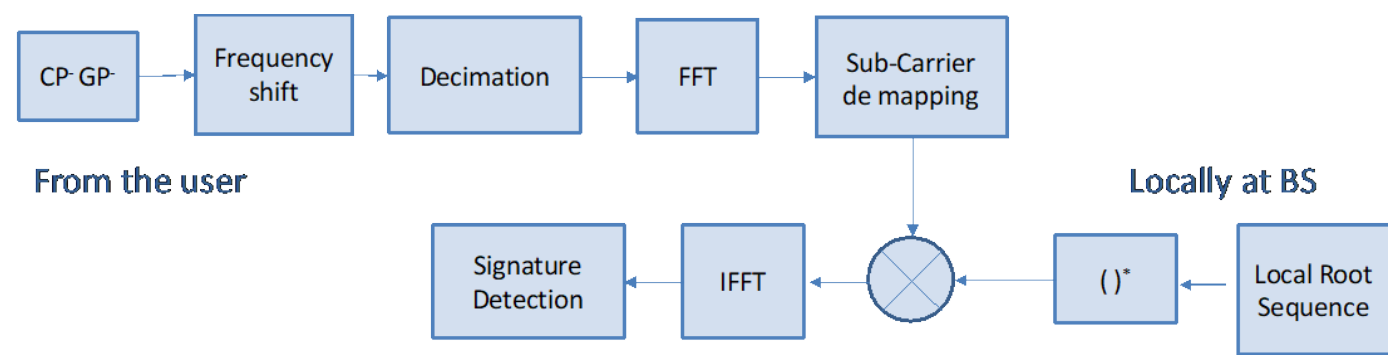

Figure 2 PRACH receiver block diagram. 


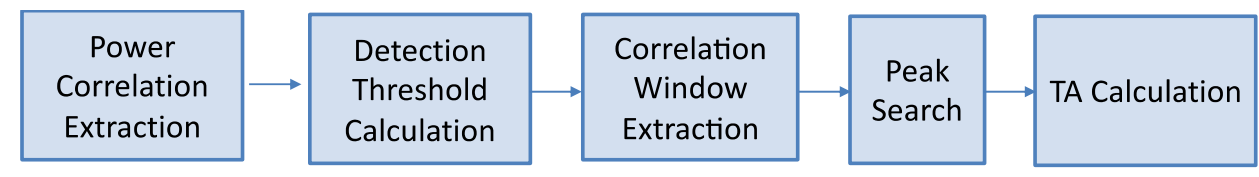

Figure 3 Detection algorithm block diagram

different physical root. In addition, it shows an acceptable resilience to frequency offset, which is a very useful property in scenarios with high mobility.

\section{CONVENTIONAL PRACH DETECTION}

The classical approach followed in previous works, i.e. [2]-[5], to deal with PRACH detection problem is to cast it in a test of hypothesis framework, where the value of the threshold is calculated by considering the statistics of the signal and the missed detection and false alarm probabilities. The threshold is then used to compare the samples of the PDP associated with the received signal to decide the presence or the absence of the preamble. The PRACH receiver must be implemented in order to maximize the correct preamble detection probability and minimize the delay due to the processing. The block diagram of the conventional PRACH receiver, which shares some operations with the Orthogonal Frequency Division Multiplexing (OFDM) demodulator, is illustrated in Fig. 2. The preamble signal is extracted after CP and Guard Period (GP) removal, down-sampling, application of Fast implementation of discrete Fourier Transform (FFT), sub-carrier selection, and correlation in the frequency domain with the local ZC root sequence [8].

The detection algorithm is implemented in the last block of Fig. 2, whose content is shown in Fig. 3. The classical detection approach starts from the computation of the average

$$
m_{\text {tot }}=\frac{1}{N_{\text {sample }}} \sum_{k=0}^{N_{\text {sample }}-1} p d p(k),
$$

where $p d p(k)$ is the discrete-time PDP obtained by taking the magnitude square of the time-domain samples at the output of the inverse FFT (IFFT) block. The power threshold below which we assume to have only noise is

$$
T_{\text {total }}=T_{\text {th }} m_{\text {tot }}
$$

where $T_{t h}$ is the optimal threshold computed from the theory, which is computed according to a general probability of false alarm defined as [7]

$$
P_{f a}=1-\left(1-\exp \left(-N_{a} \cdot N_{n c a}-1\right)\right) \sum_{k=0}^{N_{a} \cdot N_{n c a}-1} \frac{1}{k !}\left(N_{a} N_{n c a} T_{t h}\right)^{k},
$$


where $N_{a}$ is the number of antennas and $N_{n c a}$ is the number of non-coherent accumulations, which accounts for possible sequence repetitions, such that $N=N_{a} \cdot N_{\text {nca }}$ turns out to be the overall Degree-of-Freedom (DoF). The above formula holds for a complex Additive White Gaussian Noise (AWGN) channel where, in the absence of the desired signal, the power envelope is a central Chisquare distribution whose DoFs are determined by the number of Gaussian random variables added together [7]. Extending this concept to complex samples, the DoFs are doubled because these are the sum of two different random signals. Moreover, since the received sequence is defined as the sum of the streams received by each of the two antennas, the resulting DoFs of the PRACH include also the number of received antennas [7]. The indexes of the PDP below the threshold $T_{\text {total }}$ given in (4), i.e. $\mathcal{K}=\left\{k_{n_{1}}, k_{n_{2}}, \ldots, k_{N_{\text {noise }}} \mid p d p(k)<T_{\text {total }}\right\}$, define the samples that are considered as noise. From these samples, it is possible to calculate the average power of the noise as

$$
m_{n}=\frac{1}{N_{\text {noise }}} \sum_{k \in \mathcal{K}} p d p_{\text {noise }}(k) .
$$

Then, the threshold used to detect the presence of the preamble is

$$
T_{\text {det }}=T_{t h} m_{n}
$$

Finally, the method exploits the property of the ZC sequence and implements a window-based detection approach, where the size of the window is the same as that of the ZCZ, i.e. $N_{c s}$ [7]. According to this approach, the presence or the absence of a preamble is verified for every window by comparing each sample inside the window with the detection threshold level $T_{\text {det }}$. The maximum value in the window that is higher than $T_{\text {det }}$ locates the position of the candidate preamble. Its position inside the window is used to estimate the TA by using an interpolation procedure. In this paper, we refer to this approach as one-step PRACH detection algorithm.

\section{ERROR EVENTS}

In contrast to previous works, and in agreement with 3GPP 36.104 specifications, in this paper the performance is studied with reference to the combined effect of the following different probabilities of error:

1. $P_{d}(E)$ defines the probability of missed detection of the preamble. This error occurs when the preamble is sent by the user, but it is not detected by the BS due to an incorrect estimation of 
$T_{d e t}$ in (7) or to the presence of multipath attenuation. In this way a new RACH procedure needs to be performed.

2. $P_{w}(E)$ defines the probability of detection of a preamble other than the one sent when only one peak is above $T_{d e t}$. It means that the preamble is sent by the user, but the BS incorrectly chooses another one. In this way the BS starts to allocate resources for that incorrectly detected user.

3. $P_{s p}(E)$ defines the probability of detecting more than one peak above $T_{\text {det }}$ in the same correlation window. In this situation it could happen that the peak associated with the PRACH preamble is not the highest since it was received with large attenuation from the channel. The meaning of this will be better explained in the next section.

4. $P_{t a}(E)$ defines the probability of wrong TA estimation given that a preamble has been detected. It means that the preamble is sent by the user and detected by the BS. However, during the interpolation procedure to estimate the TA, the BS does not correctly define the position of the user inside the cell. So, it needs to wait for another message to correctly estimate it.

The sum of the first three error events described above, which are mutually exclusive, gives the total missed detection probability:

$$
P_{m d}(E)=P_{d}(E)+P_{w}(E)+P_{s p}(E) .
$$

It is important to underline that these three probabilities of error are related to $P_{t a}(E)$, since the TA estimation starts after the preamble detection procedure (see Fig. 3) and so this is the main reason for which we also consider $P_{t a}(E)$ as a tool to evaluate the goodness of our design. According to 3GPP specifications [6], the term $P_{t a}(E)$ should be also considered in the Right-Hand Side (RHS) of (8). In our results we will include its contribution.

In this paper we consider a more practical approach compared to classical ones, where the contribution of each term in the RHS of (8) is evaluated separately. Considering each term individually allows us to show the main weaknesses of previous approaches and this is the main reason that motivated us to propose a new procedure to reduce both $P_{w}(E)$ and $P_{s p}(E)$ from a practical point of view, leading to an improvement of the PRACH receiver performance. 


\section{ISSUES OF THE ONE-STEP PRACH DETECTION}

The one step PRACH detection algorithm is essentially a frequency-domain correlation method. It is worth observing that the higher is the Signal-to-Noise Ratio (SNR), the lower is $m_{N}(6)$ and, therefore, the detection threshold (7). In this situation several false peaks were observed, as shown in Fig. 4. These undesired detections are called "side peaks", which are very critical at high SNR values and affect the total missed detection $P_{m d}(E)$ probability by increasing the contributions of $P_{s p}(E)$ and $P_{w}(E)$ in (8). This phenomenon happens because the value of the threshold is too low.

Hence, a possible solution to deal with this issue is to increase the value of the threshold when the following condition is satisfied:

$$
\frac{\beta \max _{k} p d p(k)}{T_{t h} m_{n}}>1 .
$$

In fact, the ratio $\max _{k} p d p(k) / m_{n}$ can be considered as an estimate of the peak SNR. The parameter $\beta$ in (9), must be set to satisfy the 3GPP conformance requirements [6]. In particular, it is empirically computed to distinguish the low from the high SNR condition.

As we will see, the choice of $\beta$ and the correction of the threshold, which lead to a two-step procedure, are not enough to satisfy the 3GPP requirements. The power accumulated on the side peaks due to the presence of multipath, e.g. in case of an Extend Terrestrial Urban (ETU) [6] environment, can exceed the threshold computed in (7) by using the average noise power, leading to an incorrect choice of the peak, which increases $P_{s p}(E)$, and a wrong estimation of the TA, which

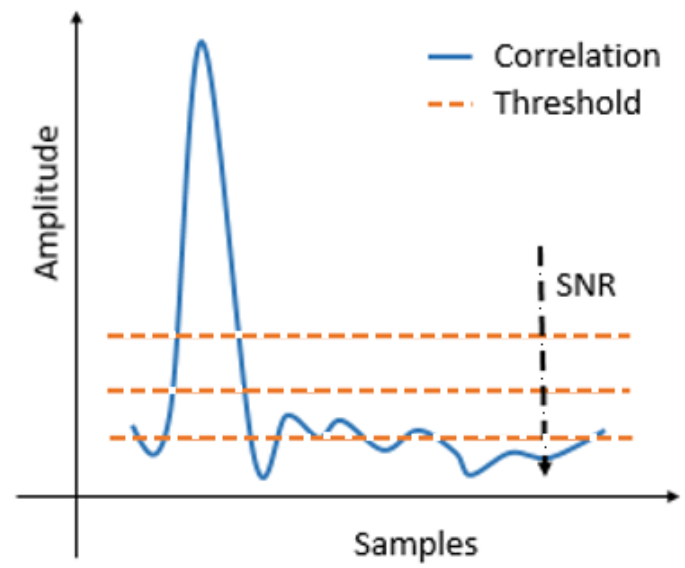

Figure 4 Undesired peaks vs SNR 
increases $P_{t a}(E)$. To manage this other issue, we propose to add another step to the detection that leads to the proposed three-step PRACH detection algorithm described in the next section.

\section{PROPOSED Three-step AlgorithM}

In this section we illustrate the proposed algorithm, where the problem of finding the correct peak and its position inside the window is addressed. The study is done considering the PRACH parameters proposed in the 36.104 specifications as a reference but demonstrated by simulations, which does not affect the generality of the discusssion.

First, we found empirically the value of $\beta$ in (9). In fact, if (9) is verified in the considered searching window, the threshold is updated as:

$$
\text { if (9) then } T_{\text {det }}^{\text {updated }}=\beta \max _{k} p d p(k) \text {. }
$$

The choice of $\beta$ takes into account that a correction of the threshold, which tries to mitigate the detection of a false peak, could have as a side effect an increase of $P_{d}(E)$. In fact, an excessive increase of the threshold could lead to the event where the peak generated by the effective presence of a preamble is not detected. Starting from this observation, there are two possible error cases:

1. an error conditioned to the correction of the threshold $P(E \mid$ correction $)$;

2. an error conditioned to the non-correction of the threshold $P(E \mid$ non - correction $)$.

The term $P(E \mid$ correction $)$ is the probability of making an error given that the threshold has been corrected while $P(E \mid$ non - correction $)$ is the probability of making an error given no correction occurrence. Both the two conditional error probabilities were estimated empirically by means of computer simulations. After many trials it was ascertained that a good choice for $\beta$ is 0.0315 . The results for different SNR conditions, considering the ETU channel, are reported in Table 1.

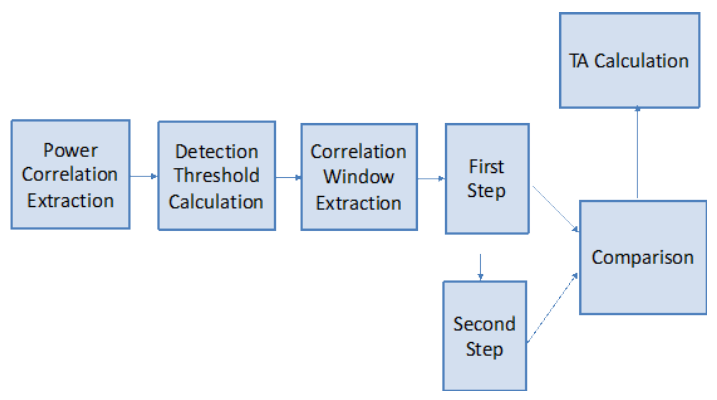

Figure 5 Multi-steps detection algorithm 
As can be observed, threshold-based SNR adaptation performs better than the case in which we never update the threshold, meaning that the value $\beta$ has been chosen correctly. Anyway, this approach does not always guarantee a null conditioned probability of error, i.e. the correction of the threshold itself generates some errors. To solve this issue the proposed approach is based on the idea of filtering the PDP sequence, as will be explained below in step 2 of the algorithm.

Table 1. Conditioned probability of error after threshold correction (right) and non-correction (left) for different SNR values for $\boldsymbol{\beta}=0.0315$.

\begin{tabular}{|c|c|c|}
\hline \multicolumn{2}{|c|}{ Probability of error } \\
\hline SNR (dB) & $\begin{array}{c}\text { Without threshold } \\
\text { correction }\end{array}$ & $\begin{array}{c}\text { After threshold } \\
\text { correction }\end{array}$ \\
\hline $\mathbf{- 2 0}$ & 0.0001 & 0 \\
\hline $\mathbf{- 1 8}$ & 0.0011 & 0 \\
\hline $\mathbf{- 1 6}$ & 0.001 & 0 \\
\hline $\mathbf{- 1 4}$ & 0.0011 & 0 \\
\hline $\mathbf{- 1 2}$ & 0.0008 & 0 \\
\hline $\mathbf{- 1 0}$ & 0.0005 & 0 \\
\hline $\mathbf{- 8}$ & 0.00132 & 0.00118 \\
\hline $\mathbf{- 6}$ & 0.00164 & 0.00112 \\
\hline $\mathbf{- 4}$ & 0.00314 & 0.00138 \\
\hline $\mathbf{- 2}$ & 0.00568 & 0.00178 \\
\hline $\mathbf{0}$ & 0.01473 & 0.00102 \\
\hline $\mathbf{2}$ & 0.03648 & 0.00136 \\
\hline $\mathbf{4}$ & 0.07692 & 0.00141 \\
\hline $\mathbf{6}$ & 0.23529 & 0.0011 \\
\hline $\mathbf{8}$ & 0.2 & \\
\hline & & 0 \\
\hline
\end{tabular}

This allows us to reduce the effect of the multipath channel and, at the same time, to increase the accuracy of TA estimation. The resulting algorithm is illustrated in Fig. 5. Detailed steps are as follow:

1. In the first detection step, the proposed approach processes, one after the other, small intervals of the entire PDP. The lengh of each interval is obtained from

$$
L=\frac{N_{I F F T}}{N_{u}-1}+1
$$


where $N_{I F F T}$ is the size of the IFFT to generate the PDP in time domain and $N_{u}$ is equal to $\left\lfloor\frac{N_{z c}}{N_{c S}}\right\rfloor-1$ (see Sec. III). This is an adaptation of the ZCZ length $N_{c s}$ after the zero padding and IFFT algorithm. Each interval is denoted as window $_{k}$, for $k=0: L: N_{I F F T}-1$. For each window ${ }_{k}$, the threshold is calculated using (10), the position and the value of the peak in the considered interval is determined as:

$$
\operatorname{peak}_{k}=\max \left(p d p\left(\text { window }_{k}\right)\right), \quad \text { for } k=0: L: N_{I F F T}-1
$$

2. In this step we follow the same approach as that proposed in [9], where a filtering operation is introduced if the peak value is greater than $T_{\text {det }}$. The filtering operation is done in the considered window $_{k}$ with the goal of reducing the effect of the delay spread. To this aim, a moving average filter is applied [9]. As we can see in Figs. 6 and 7, the effect of the moving average filter on the 1024 samples of the PDP is that of reducing the side peaks introduced by the multipath scenario. Since the searching window has dimension less than $N_{C S}$, the length of the filter could not be too long otherwise the TA estimation, which is performed in the next step, is affected. The length of this filter, found after several simulations, is:

$$
M=\left\lceil\frac{N_{I F F T}}{N_{z c}} c\right\rceil
$$

where $c$ is a constant value, in our case equal to 5 , and \lceil\rceil is the lowest integer greater than its argument.

In the filtered window, i.e. $\operatorname{window}_{k}$, the position and the value of the peak, i.e. $p e a k^{\prime}{ }^{\prime}$, is determined and compared with the given threshold.

$$
\begin{aligned}
& \text { if } \text { peak }_{k}>T_{\text {det }} \\
& \text { window }_{k}^{\prime}=\text { window }_{k} * \operatorname{rect}\left(\frac{k}{M}\right) M \\
& \text { peak }_{k}^{\prime}=\max \left(\text { pdp }\left(\text { window }_{k}{ }_{k}\right)\right)
\end{aligned}
$$

where $*$ denotes convolution and $\operatorname{rect}(k)=\left\{\begin{array}{cc}1, & -\lceil(M-1) / 2\rceil \leq k \leq\lfloor(M-1) / 2\rfloor \\ 0, & \text { otherwise }\end{array}\right.$. 


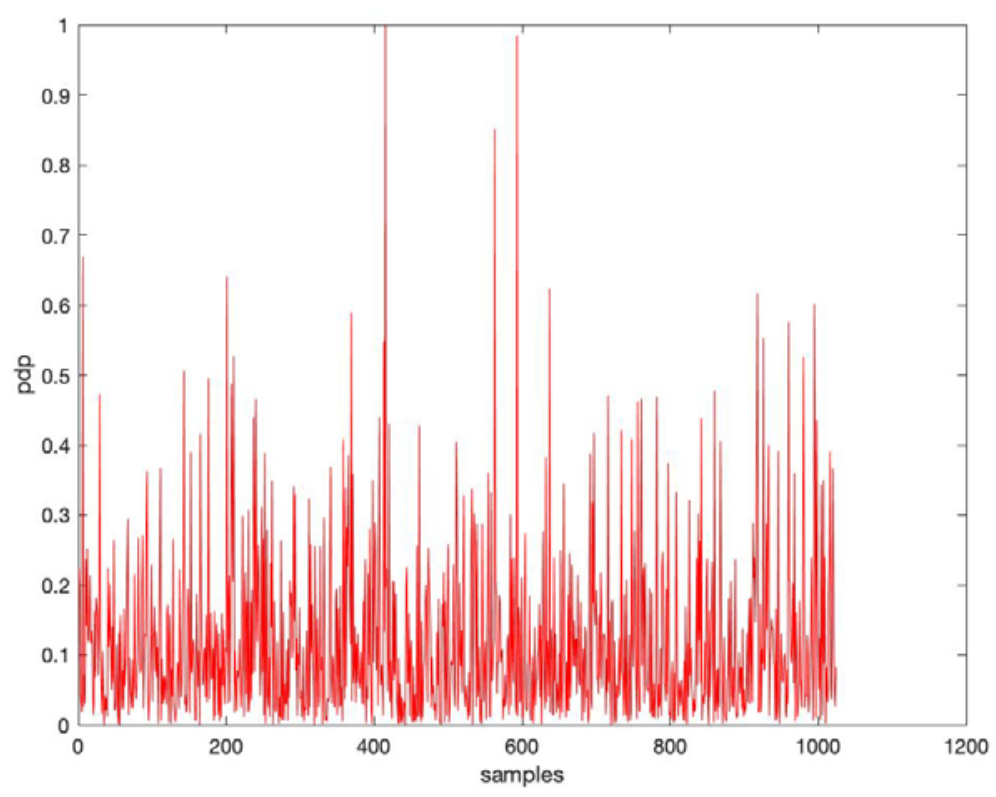

Figure 6 Normalized PDP vs. time samples.

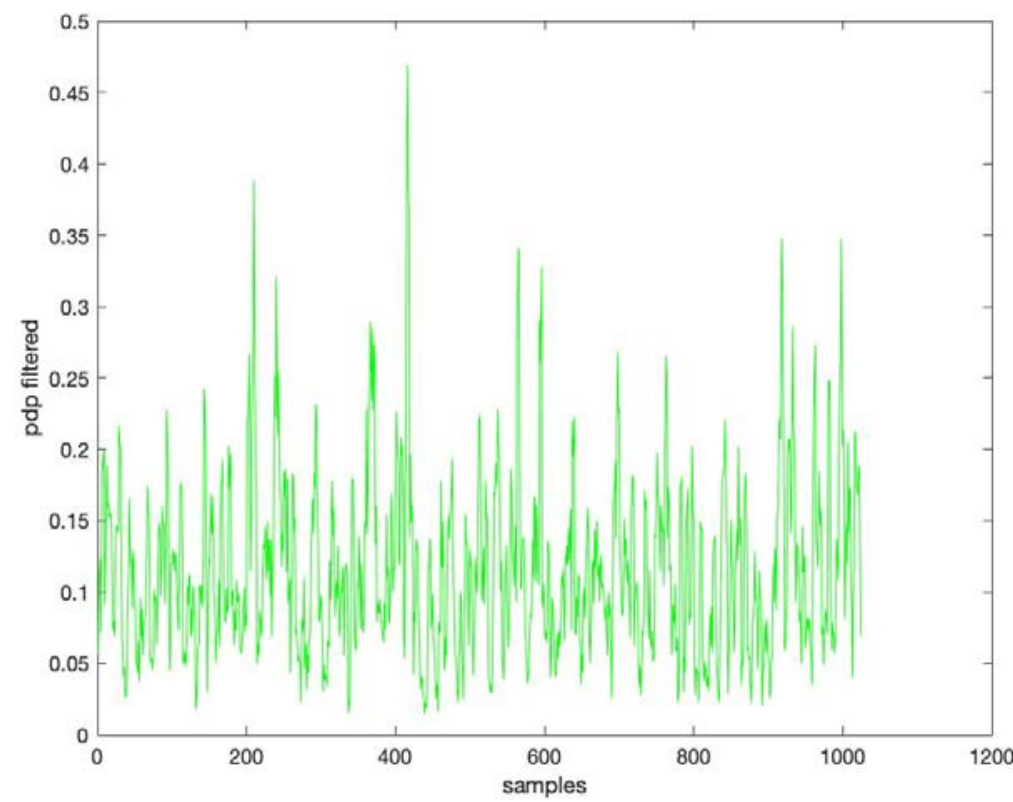

Figure 7 Normalized and filtered PDP vs. time samples.

3. In the third, and last, step it is decided which detection is the most reliable between the two steps, checking if:

$$
M \text { peak }_{k}^{\prime}>\text { peak }_{k} \text {. }
$$

Note that, the decision if a correct detection has happened or not is different in low and in high SNR scenario: 
a. in the low SNR scenario, a detection occurs if at least in one of the two steps there is a crossing of the threshold: in this case its information is taken as correct;

b. in the high SNR scenario, a detection occurs if in both the steps there is a crossing of the threshold. This is done to reduce the error detection due to side peaks. At this point, the peak with the highest amplitude is considered as the most reliable one.

Another important aspect is that with the introduction of the filtering operation in the second step, the $P(E \mid$ correction $)$ goes to zero, with an order of magnitude less than $10^{-4}$ for all the SNR values considered in Table 1. At this point, the delay offset is calculated, and the TA is updated considering the selected peak position.

The proposed algorithm, in case of correct preamble detection, takes into account the reliability of the estimated TA, and how it affects the missed detection probability as suggested by the 3GPP specifications [6]. The latter is achieved by calculating $P_{t a}(E)$, which is the number of times for which the algorithm correctly finds the preamble but overestimates the TA, leading to an error during the set-up procedure. The achieved results are shown in Table 2. As it can be seen $P_{t a}(E)$ is more or less constant for all the SNR values considered.

Table 2. Probability of wrong estimated TA for different SNR values.

\begin{tabular}{|c|c|c|c|c|c|c|c|}
\hline SNR (dB) & $P_{t a}(E)$ & SNR (dB) & $P_{t a}(E)$ & SNR (dB) & $P_{t a}(E)$ & SNR (dB) & $P_{t a}(E)$ \\
\hline $\mathbf{- 2 0}$ & 0.0011 & $\mathbf{- 1 2}$ & 0.0033 & $\mathbf{- 4}$ & 0.0030 & $\mathbf{4}$ & 0.0022 \\
\hline $\mathbf{- 1 8}$ & 0.0013 & $\mathbf{- 1 0}$ & 0.0021 & $\mathbf{- 2}$ & 0.0019 & $\mathbf{6}$ & 0.0020 \\
\hline $\mathbf{- 1 6}$ & 0.0022 & $\mathbf{- 8}$ & 0.0030 & $\mathbf{0}$ & 0.0030 & $\mathbf{8}$ & 0.0020 \\
\hline $\mathbf{- 1 4}$ & 0.0026 & $\mathbf{- 6}$ & 0.0021 & $\mathbf{2}$ & 0.0020 & $\mathbf{1 0}$ & 0.0021 \\
\hline
\end{tabular}

VII. NUMERICAL RESULTS

In this section the performance of the proposed approach is reported and compared with that of the one step algorithm. Presented numerical results were obtained by using Monte Carlo simulations. Computer simulations were done using MATLAB code. For each value of SNR, a number higher than 10000 runs was done to have a high accuracy of the missed detection probability. In each trial the values of the noise sample were random generated with a given variance, while the parameter of the ETU channel, such as channel gain, channel delays, etc. The most relevant simulation parameters are reported in Table 3, which were taken from the scenarios defined in [6]. In what follows it is not showed any result for the false alarm probability since it is always below the value of $10^{-3}$ for both 
the channel scenarios, thus satisfying TS 36.104 specifications. This is due to the fact that the threshold is chosen according to the desired false alarm probability given in (5). Results for other bandwidths are not shown because they are very similar to those we have reported here. The missed detection probability versus SNR for the single and multi-step detector is reported in Figs. 8 and 9 for the AWGN and the ETU scenarios, respectively.

Table 3. Parameters used in computer simulations for evaluation of performance in PRACH detection.

\begin{tabular}{|c|c|}
\hline Parameter & Value \\
\hline $\begin{array}{c}\text { System } \\
\text { Bandwidth }\end{array}$ & $20 \mathrm{MHZ}$ \\
\hline PRACH Format & 0 \\
\hline Channel & AWGN/ETU70 \\
\hline Doppler & $0 / 200[\mathrm{HZ}]$ \\
\hline RX Antenna & 2 \\
\hline $\boldsymbol{N}_{\boldsymbol{z c}}$ & 839 \\
\hline $\boldsymbol{P}_{\boldsymbol{f a}}$ & 0.001 \\
\hline
\end{tabular}

In Fig. 8, both the classical one-step and the proposed three step allow to satisfy the missed detection probability required by 3GPP for AWGN channels corresponding to $10^{-2}$ at $S N R=-14.2 \mathrm{~dB}$, which is represented by the red asterisk. It is worth observing that a wrong selection in the design of the key factors could lead to a worse performance for this scenario, since the AWGN channel is only one tap channel. Threfore, a trade-off between the two different scenarios must be done in the selection of the key parameters.

On the other side for the ETU channel, the proposed detection algorithm allows to satisfy the stringent requirements defined by 3GPP in [6] that requires a missed detection probability of $10^{-2}$ at SNR=-8 dB. In fact, Fig. 9 allows us to numericaly demonstrate:

i. the rightness of the error events introduced in Sec. IV;

ii. the limitations of the one-step procedure in this region, which is manifested by the presence of an error floor; 


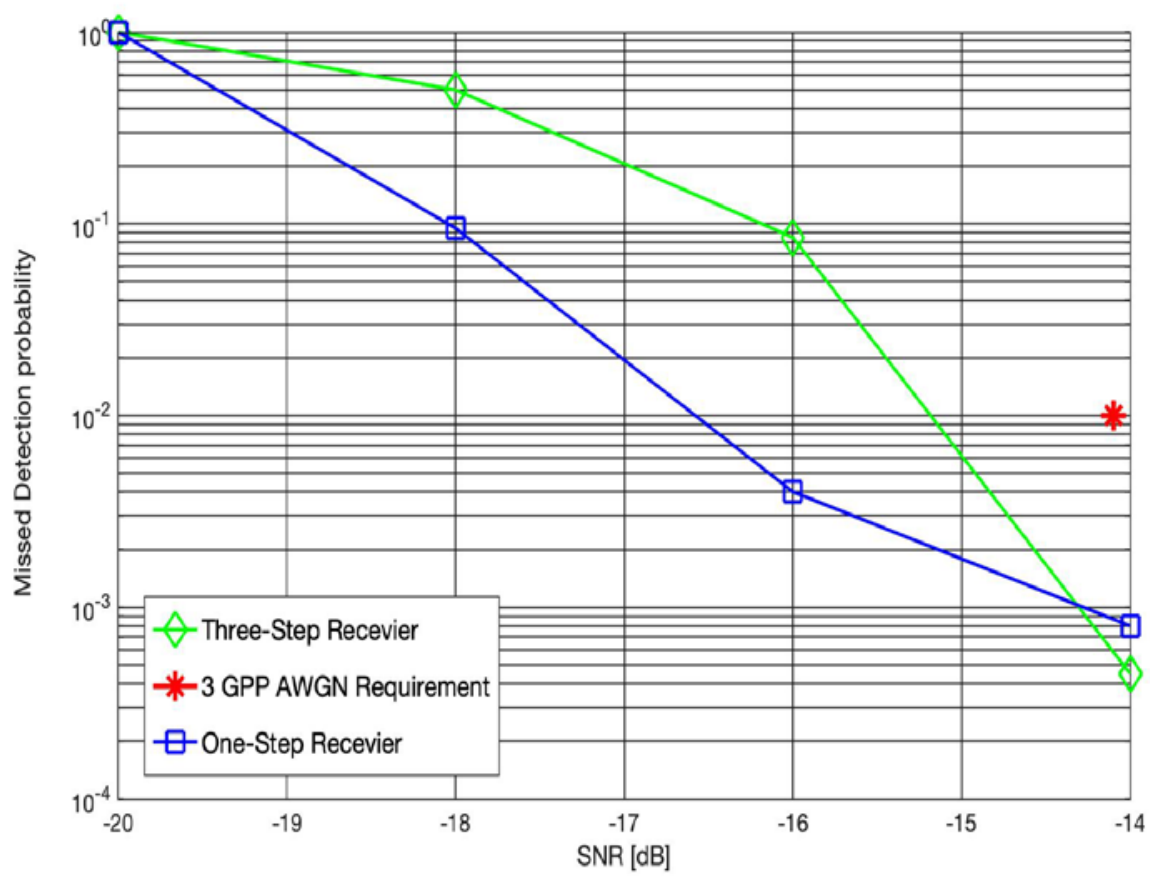

Figure 8. Probability of missed detection vs. SNR in case of transmission over the AWGN channel.

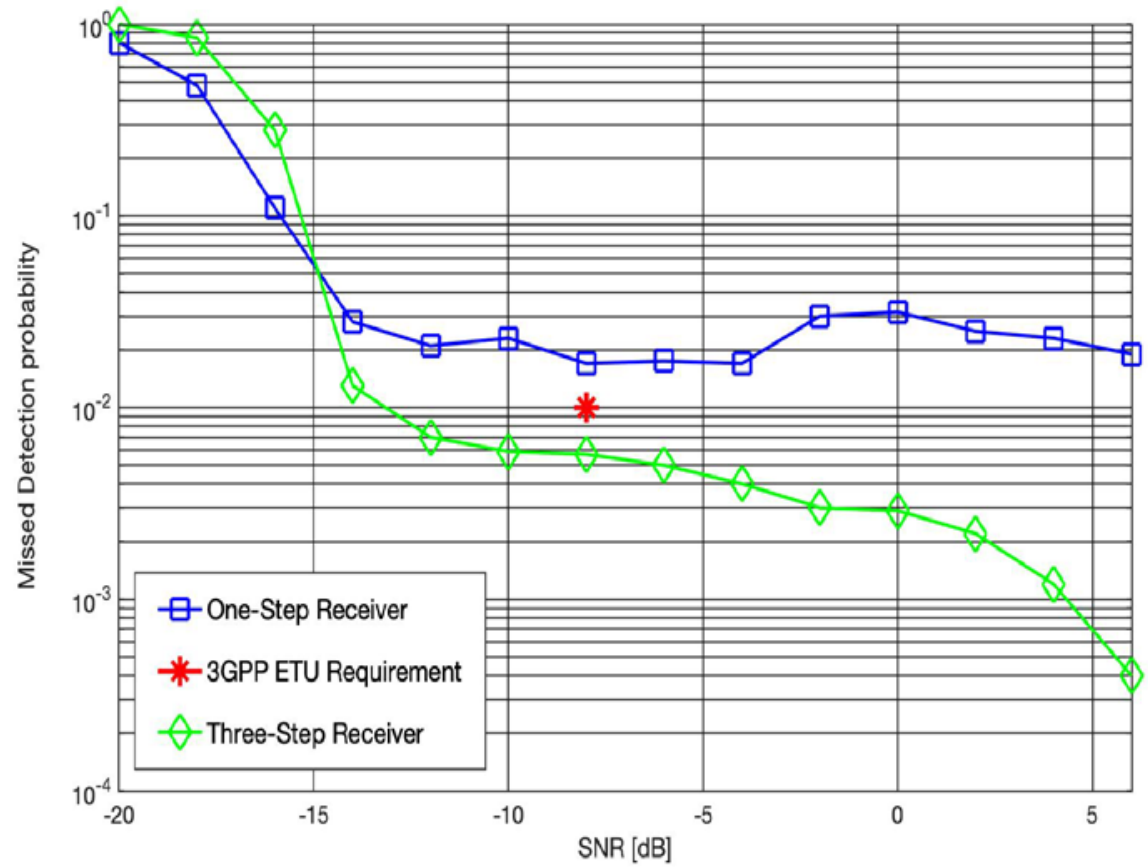

Figure 9. Probability of missed detection vs. SNR in case of transmission over the 3GPP ETU channel. 
iii. the effectiveness of the three steps method proposed in Sec. VI to mitage the error events: the trend of missed detection probability of this new approach is consistent in the entire range of considered SNR values and allows to meet, with a slight buffer, the stringent 3GPP requirements.

\section{PRACH PREAMBLES IN 5G NR}

As in LTE, 5G NR is also based on the RACH procedure described Fig. 1 [10]. The novel aspect in 5G NR is the introduction of two types of preambles, which are referred to as the long and the short preamble, respectively [11]. As the name suggests, the two preambles differ in their length and in the numerology of the parameters used in the transmission, e.g. subcarrier spacing, bandwidth, etc. The type of preamble is part of the cell random-access configuration broadcast information and it is unique within a cell. The long preambles partly originate from the preambles used for LTE randomaccess [11]. They are based on a sequence length of 839 samples and a subcarrier spacing of either $1.25 \mathrm{kHz}$ or $5 \mathrm{kHz}$ for frequency bands below $6 \mathrm{GHz}$ [10]. For this case, our approach can be directly applied since there are not significant differences in the parameters given in Table 3 used in the simulations for LTE. The short preambles are, in general, shorter than the long preambles in terms of number of OFDM symbols. Therefore, in most of the cases it is possible to have multiple preamble transmissions multiplexed in time within a single RACH slot. In other words, for short preambles there may not only be multiple occasions of detection in the frequency domain but also in the time domain within a single resource slot [11]. In this case the application of the proposed approach should be modified to take into account the repetition in time of short preambles in a slot, allowing for a possible improvement in the detection performance due to the exploitation of inherent diversity introduced by the repetition itself.

\section{CONCLUSION}

This paper has focused on the characterization, description, and demonstration of the issues associated with error events arising in the classical detection of the PRACH signal in the uplink of wireless cellular network. We have developed a new three-step PRACH detector that overcomes the classical one step approaches and faces with their problems, thus allowing us to satisfy the 3GPP requirements for both the idea AWGN channel and frequency selective ones, i.e. ETU. In fact, the 
classical approach does not take into account the effect of the channel itself on the preamble transmission, showing a performance degradation in case of frequency selective channels. Computer simulations demonstrate the effectiveness of the proposed solution in the case of time dispersive channels. Moreover, the suggested method together with the given characterization of the error events could be the basis to propose more advanced solutions with different degrees of complexity. The proposal, originally designed and tested for last generation of the $4 \mathrm{G}$ wireless cellular systems, can be directly extended to 5G. A research direction that will be investigated is the extension to 5G of the proposed three step procedure by considering its appropriateness to different use cases.

\section{ACKNOWLEDGEMENT}

All the simulations were performed in the Lab of Azcom srl, Rozzano, Italy, where real data have been used for testing the proposed approach. This had an important role to obtain the presented results because allowed us to properly justify constant and empirical values introduced in Sec. VI and chosen after several computer simulations.

\section{REFERENCES}

[1] R. Cheng, Z. Becvar, Y. Huang, G. Bianchi, and R. Harwahyu, “Two-phase random access procedure for LTE-A networks,” IEEE Transactions on Wireless Communications, vol. 18, no. 4, pp. 2374-2387, April 2019.

[2] C. Yu, W. Xiangming, Z. Wei, and L. Xinqi, "Random access algorithm of LTE TDD system based on frequency domain detection,” in Proc. of Fifth International Conference on Semantics, Knowledge and Grid (SKG), Zhuhai, China, 2009, pp. 346-350.

[3] S. Kim, K. Joo, and Y. Lim, “A delay-robust random access preamble detection algorithm for LTE system,” in Proc. of IEEE Radio and Wireless Symposium (RWS), Santa Clara, CA, 2012, pp. 75-76.

[4] M. M. Mansour, “Optimized architecture for computing Zadoff-Chu sequences with application to LTE,” in Prof. of IEEE Global Telecommunications Conference (GLOBECOM), Honolulu, HI, 2009, Pp. 1-6.

[5] L. Wu, X. Zhang, and Y. Yang, “Design and realization of baseband signal downsampling in LTE system,” International Journal of Future Generation Communication and Networking, pp. 81-88, 2013.

[6] 3GPP, “3rd Generation Partnership Project; Technical Specification Group Radio Access Network NR Base Station (BS) radio transmission and reception, 36.104”, 2018.

[7] S. Sesia, M. Baker, and I. Toufik, “LTE - The UMTS long term evolution: from theory to practice,” John Wiley \& Sons, 2011. 
[8] 3GPP, “Technical Specification Group Radio Access Network; Evolved Universal Terrestrial Radio Access (E-UTRA); Physical channels and modulation, 36.211”, 2018.

[9] Y. Zhang, Y. Liu, and Z. Zhang, "Simulation and implementation of PRACH signal detection in LTE-A,” Journal of Computer Applications, pp. 1-7, Feb. 2018.

[10] E. Dahlman, S. Parkvall, and J. Sköld, “4G LTE-advanced pro and the road to 5G.” Accademic Press, 2016.

[11] E. Dahlman, S. Parkvall, and J. Sköld, “5G NR: The next generation wireless access technology.” Accademic Press, 2018. 